\title{
Local electrical and dielectric properties of nanocrystalline yttria-stabilized zirconia
}

\author{
N. H. Perry · S. Kim · T. O. Mason
}

Received: 21 December 2007 / Accepted: 12 February 2008/Published online: 30 April 2008

(C) Springer Science+Business Media, LLC 2008

\begin{abstract}
Grain core and grain boundary electrical and dielectric properties of nanocrystalline yttria-stabilized zirconia (YSZ) were analyzed using a novel nano-Grain Composite Model (n-GCM). Partially sintered pellets with average grain sizes ranging from 10 to $73 \mathrm{~nm}$ were analyzed over a range of temperatures using AC impedance spectroscopy (AC-IS). Local grain core and grain boundary conductivities, grain boundary dielectric constants, and effective grain boundary space charge widths were determined from the fitted circuit parameters. Required grain core dielectric constant data were provided by AC-IS measurements of single crystal YSZ over a range of temperatures. The local grain core conductivity of all the nanocrystalline samples was slightly decreased with respect to that of microcrystalline YSZ. Conversely, the local grain boundary conductivity was enhanced up to an order of magnitude compared to microcrystalline YSZ. At the nanoscale, there was a noticeable increase in local grain boundary dielectric constant versus single crystal values at the same temperature.
\end{abstract}

\section{Introduction}

Yttria-stabilized zirconia (YSZ) is the workhorse electrolyte in electrochemical devices, including solid oxide fuel cells (SOFCs), oxygen pumps, and oxygen sensors [1].

N. H. Perry · T. O. Mason $(\bowtie)$

Department of Materials Science and Engineering, Northwestern University, 2220 Campus Drive, Evanston, IL 60208, USA

e-mail: t-mason@northwestern.edu

S. Kim

Department of Chemical Engineering and Materials Science, University of California, Davis, Davis, CA 95616, USA
Much recent research has focused on enhancing the transport properties of YSZ at low temperatures to improve the commercial viability of SOFCs [2,3]. By operating at lower temperatures $\left(<600{ }^{\circ} \mathrm{C}\right)$ problems associated with deleterious interfacial reactions, differential thermal expansion, and gas sealing can be ameliorated. Also, less expensive interconnect materials (e.g., stainless steel) can be employed. To improve the transport properties, researchers have investigated nano-scale YSZ electrolytes, where the grain size and/or film thickness is $<100 \mathrm{~nm}$.

There are conflicting reports concerning the grain sizedependence of transport properties in nano- versus microscale YSZ. In a recent review, Guo and Waser noted that, for several acceptor-doped zirconias, the specific grain boundary conductivity increases as the grain size decreases into the nanocrystalline regime, while the grain core conductivity decreases slightly [1]. Mondal et al. [4] reported enhanced specific grain boundary conductivities in YSZ of grain size $25-50 \mathrm{~nm}$, at doping levels of 1.7 and $2.9 \mathrm{~mol} \%$ $\mathrm{Y}_{2} \mathrm{O}_{3}$. Mandani et al. [5] reported higher grain boundary conductivity than grain core conductivity in $4 \mathrm{~mol} \% \mathrm{Y}_{2} \mathrm{O}_{3^{-}}$ doped $\mathrm{ZrO}_{2}$, but not at the 9 mol\% level. Verkerk et al. [6] found increasing specific grain boundary conductivity with decreasing grain size in microcrystalline $8 \mathrm{~mol} \% \quad \mathrm{Y}_{2} \mathrm{O}_{3^{-}}$ doped $\mathrm{ZrO}_{2}$, but only at large grain sizes. The most dramatic result was that of Kosacki et al. [7], who showed a 1-2 order of magnitude increase in the total conductivity in spin-coated YSZ thin films with $\sim 20 \mathrm{~nm}$ grain size versus a polycrystalline bulk sample with large grains $(2.4 \mu \mathrm{m})$. Karthikeyan et al. [8] reported an increase of conductivity up to one order of magnitude in e-beam deposited YSZ thin films as the film thickness was reduced to $17 \mathrm{~nm}$. In contradistinction to these studies, Guo et al. [9] saw a slight decrease in the conductivity of $12 \mathrm{~nm}$ and $25 \mathrm{~nm}$ films compared to bulk polycrystalline values. 
The aforementioned papers relied upon the brick-layer model (BLM) to extract grain boundary properties (e.g., the "specific grain boundary conductivity") and grain core properties. We recently demonstrated that the BLM is no longer valid when the grain size is reduced into the nanocrystalline regime, i.e., at grain sizes $<100 \mathrm{~nm}[10,11]$. The present work employs our recently developed "nanoGrain Composite Model" or n-GCM to extract local grain boundary and grain core properties for nano- versus microcrystalline YSZ.

\section{The nano-grain composite model (n-GCM)}

Extracting local properties (grain core, grain boundary) from AC impedance spectroscopy (AC-IS) measurements requires a representative microstructural model and a corresponding equivalent circuit. The original equivalent circuit used to describe YSZ and microcrystalline electrolytes in general was developed by Bauerle [12], for ceramics with resistive grain boundaries and conductive grain cores. This simple boundary-layer model was further developed by Beekmans and Heyne [13] and was labeled as the "brick-layer model" or BLM by Burggraaf and coworkers $[6,14]$. The equivalent circuit consists of two RC parallel elements in series, one representing the grain cores and the other representing the grain boundaries. In the well-known Boukamp notation [15], this circuit is represented as (RC)(RC). For microcrystalline electroceramics, with thin and less conductive grain boundaries (vis-à-vis grain core values), the BLM is quite appropriate [10,11].

In the nanoscale regime, however, the BLM is no longer reliable, owing to the contribution of grain boundary conduction parallel to the direction of current flow. Näfe [16] developed a modified BLM by connecting the central grain core/grain boundary serial path in parallel with the sidewall grain boundary path. This series/parallel BLM, or SPBLM, was applied to the analysis of AC-IS measurements on nanocrystalline $\mathrm{CeO}_{2}$ [17]. We subsequently demonstrated, however, that the SP-BLM also fails in the nanocrystalline regime $[10,11]$.

To correct the deficiencies in the BLM and variants in the nanocrystalline regime, we recently developed a "nanoGrain Composite Model" or n-GCM [10, 11]. First, a 3D pixel-based computer representation was developed for the BLM, taking into account the true 3D current distribution in the brick-layer structure. This model, referred to in our prior work as the "nested cube model" or NCM, accurately models the electrical and dielectric properties of the 3DBLM over the entire range of grain core volume fractions $(\phi)$ from microcrystalline $(\phi=1)$ to nanocrystalline $(\phi=0)$. Upon evaluating other microstructural and effective media models, it was demonstrated that only the NCM and the Maxwell-Wagner/Hashin-Shtrikman (MW-HS) effective medium model are capable of representing composite AC-IS behavior over all values of $\phi$ [10]. Furthermore, the predictions of the NCM and the MW-HS model are in relatively close agreement over all ranges of $\phi$ and of local properties ( $\sigma_{\mathrm{gb}}=$ grain boundary conductivity, $\varepsilon_{\mathrm{gb}}=$ grain boundary dielectric constant, $\sigma_{\mathrm{gc}}=$ grain core conductivity, and $\varepsilon_{\mathrm{gc}}=$ grain core dielectric constant). This agreement is fortuitous, since Bonanos and Lilley [18] were able to develop an (RC)(RC) equivalent circuit corresponding to the MW-HS model and a set of closed-form solutions allowing the four RC elements to be calculated from $\phi$ and the local properties $\left(\sigma_{\mathrm{gb}}, \varepsilon_{\mathrm{gb}}, \sigma_{\mathrm{gc}}\right.$, and $\left.\varepsilon_{\mathrm{gc}}\right)$. For the n-GCM, a reverse set of equations was developed to go from the four AC-IS-derived $(\mathrm{RC})(\mathrm{RC})$ values to the underlying $\left(\sigma_{\mathrm{gb}}, \varepsilon_{\mathrm{gb}}, \sigma_{\mathrm{gc}}, \varepsilon_{\mathrm{gc}}, \phi\right)$ values [11].

It should be noted that the extraction of the local properties, using the n-GCM, requires that one of the five unknown parameters $\left(\sigma_{\mathrm{gb}}, \varepsilon_{\mathrm{gb}}, \sigma_{\mathrm{gc}}, \varepsilon_{\mathrm{gc}}\right.$, and $\left.\phi\right)$ be known. It is reasonable to assume that the grain core dielectric constant will be the same as the single crystal dielectric constant at the corresponding temperature. In the present work, the dielectric constant of single crystal YSZ of comparable composition was measured versus temperature to provide the necessary data.

It should also be stressed that the n-GCM takes into account actual grain shape, i.e., that grains in polycrystalline ceramics are not cubic bricks. We were able to demonstrate that the electrical behaviors of icosahedral or dodecahedral grains fall intermediate to the nested cube and MW-HS models, and are most likely closer to the MWHS situation [11]. Furthermore, it is likely that grain core shape is nearly spherical as $\phi$ goes to zero (the nanocrystalline regime), such that the n-GCM becomes indistinguishable from the MW-HS model in this limit. In this case the n-GCM, which employs a methodology based upon the MW-HS equivalent circuit, is the most accurate representation of the electrical/dielectric structure-property relations of nanocrystalline ceramics.

\section{Experimental procedure}

Nanocrystalline YSZ $\left(8 \mathrm{~mol} \% \quad \mathrm{Y}_{2} \mathrm{O}_{3}\right)$ with an average particle size of 5-10 nm was obtained from Fuel Cell Materials (Lewis Center, OH, USA). Quoted impurity concentrations were $\mathrm{Si}, \mathrm{Ca}, \mathrm{Al}, \mathrm{Mg}$, and $\mathrm{Cr}<100$ ppm, with $\mathrm{Ca}$ sometimes slightly higher, and $\mathrm{Fe}, \mathrm{Zn}, \mathrm{Cu}$ at $<10 \mathrm{ppm}$. The powder was ground and uniaxially pressed at $125 \mathrm{MPa}$ to a green density of $40 \%$, then isostatically pressed at $270 \mathrm{MPa}$ to $50 \%$ green density, into $\sim 2 \mathrm{~mm}$ thick pellets with an average diameter of $10 \mathrm{~mm}$. The pellets were partially sintered for $1 \mathrm{~h}$ in air on a YSZ powder bed of 
identical composition to avoid contamination, with gradual heating and cooling (at $5{ }^{\circ} \mathrm{C} / \mathrm{min}$ ) to minimize thermal shock and cracking. Each pellet was sintered at a different temperature between 800 and $1,050{ }^{\circ} \mathrm{C}$, to achieve a range of grain sizes. Average grain size was determined by X-ray diffraction (XRD) peak broadening, using a WilliamsonHall plot with Jade software [19] to separate contributions to peak broadening from grain size, strain, and instrumental effects. XRD scans were carried out from 20 to $80^{\circ} 2 \theta$ on a Geigerflex diffractometer (Rigaku, Japan). Grain sizes were verified with field emission SEM (Hitachi S4800) images of fracture surfaces. All pellets were polished with diamond paste to $1 \mu \mathrm{m}$ roughness prior to electroding. Sample densities were calculated from the measured masses and dimensions (final thickness $\sim 1.7 \mathrm{~mm}$ ).

Sputtered silver electrodes $(\sim 100 \mathrm{~nm}$ thick) were employed rather than silver paint electrodes, to avoid the wicking of paint into the porous surfaces. Additionally, sputtered electrodes above a certain thickness on polished specimens have been shown to reduce spreading resistance/ gap capacitance problems associated with thin electrodes on rough surfaces [20]. Silver was chosen over gold as it reportedly exhibits better chemisorption of oxygen, and the electrode polarization arc in Nyquist plots is less significant [4]. AC-IS measurements were made over the frequency range of $13 \mathrm{MHz}$ to $10 \mathrm{~Hz}$, with a voltage amplitude of $1 \mathrm{~V}$ using an Agilent Technolgies 4192A impedance analyzer (Santa Clara, CA). The sample was held in a tube furnace, and measurements were taken over the temperature range, $250-600{ }^{\circ} \mathrm{C}$, low enough to avoid the onset of grain growth (see below). Temperature was monitored with an S-type thermocouple.

After correcting for geometry, the impedance data, consisting of two slightly depressed Nyquist arcs, were fitted to an (RQ)(RQ) Boukamp circuit using the "Equivalent Circuit" program [15], where Q stands for a constant phase element. These values were subsequently corrected for varying degrees of porosity (see below). The following procedure was implemented to extract the local properties from the raw impedance data.

To correct for geometry, the impedance data were multiplied by the specimen's geometric factor,

$Z_{\text {new }}^{\prime}=Z_{\text {original }}^{\prime} \cdot \frac{A}{L}$

$Z_{\text {new }}^{\prime \prime}=Z_{\text {original }}^{\prime \prime} \cdot \frac{A}{L}$

where $Z^{\prime}$ stands for real and $Z^{\prime \prime}$ stands for imaginary impedance, $A$ is cross-sectional area, and $L$ is specimen thickness. The modified Nyquist plots were fitted in Equivalent Circuit to give $R_{1}, Q_{1}$, and $n_{1}$ (the low frequency arc on the right) and $R_{2}, Q_{2}$, and $n_{2}$ values (the high frequency arc on the left), where $n_{1}$ and $n_{2}$ quantify the extent of arc depression (on the order of 0.9-1 for the nanocrystalline samples). The values of $C$ for each arc, $C_{1}$ and $C_{2}$, were calculated as:

$C_{i}=\left(R_{i}^{1-n_{i}} \cdot Q_{i}\right)^{\frac{1}{n_{i}}}$

The resistance $\left(R_{1}, R_{2}\right)$ and capacitance $\left(C_{1}, C_{2}\right)$ values were then corrected for porosity. Conductivities corresponding to each $\operatorname{arc}\left(\sigma_{1}, \sigma_{2}\right)$ were calculated:

$\sigma_{i}=\frac{1}{R_{i}}$

These conductivities were corrected for porosity using the Bruggeman symmetric model for 3-3 connectivity [21]:

$\sigma_{\mathrm{c}}=\sigma_{\mathrm{m}}\left(1-\frac{3}{2} f\right)$

where $\sigma_{\mathrm{c}}$ is the conductivity of the composite (YSZ and pores), $\sigma_{\mathrm{m}}$ is the conductivity of the matrix (YSZ), and $f$ is the volume fraction of pores, which were assumed to have zero conductivity. The porosity-corrected resistances $\left(R_{1}^{\mathrm{pc}}\right.$, $R_{2}^{\mathrm{pc}}$ ) were then back-calculated via Eq. 4. Porositycorrected capacitances were obtained assuming that the time constants $\left(\tau_{i}\right)$ for each arc are the same as before the porosity correction:

$R_{i}^{\mathrm{pc}} \cdot C_{i}^{\mathrm{pc}}=R_{i} \cdot C_{i}=\tau_{i}$

To extract the local parameters, the resulting values were inserted into the $\mathrm{n}-\mathrm{GCM}$ equations as follows:

$\sigma_{\mathrm{gb}}=\frac{1-\phi}{R_{1}^{\mathrm{pc}}(1+2 \phi)}$

$\sigma_{\mathrm{gc}}=\frac{-2 R_{2}^{\mathrm{pc}}(\phi-1)^{2}+R_{1}^{\mathrm{pc}} 9 \phi}{R_{1}^{\mathrm{pc}} R_{2}^{\mathrm{pc}}(1+2 \phi)^{2}}$

$\varepsilon_{\mathrm{gb}}=\frac{C_{1}^{\mathrm{pc}}(1-\phi)}{\varepsilon_{0}(1+2 \phi)}$

$\varepsilon_{\mathrm{gc}}=\frac{-2 C_{1}^{\mathrm{pc}}(1-\phi)^{2}+9 C_{2}^{\mathrm{pc}} \phi}{\varepsilon_{0}(1+2 \phi)^{2}}$

where $R_{1}^{\mathrm{pc}}, C_{1}^{\mathrm{pc}}, R_{2}^{\mathrm{pc}}$, and $C_{2}^{\mathrm{pc}}$ are now the geometry- and porosity-corrected values.

As mentioned previously, one parameter must be known to solve for the other four parameters. For the present work, it was assumed that the grain core dielectric constant $\left(\varepsilon_{\mathrm{gc}}\right)$ should be the same as the single crystal dielectric constant at the corresponding temperature. Unfortunately, literature data covering our experimental temperature range appear to be unavailable. Single crystals of YSZ $\left(7 \mathrm{~mol} \% \mathrm{Y}_{2} \mathrm{O}_{3}\right.$ ) with dimensions $10 \mathrm{~mm}$ by $10 \mathrm{~mm}$ by $1 \mathrm{~mm}$ were obtained from MTI Corp. (Richmond, CA, USA). The large faces 
were electroded with thin, porous layers of silver paste. AC-IS measurements were made in the [100] direction from $13 \mathrm{MHz}$ to $10 \mathrm{~Hz}$ over the temperature range of 20 $500{ }^{\circ} \mathrm{C}$ with the Agilent Technologies $4192 \mathrm{~A}$ impedance analyzer.

For microcrystalline samples, alternatively, the BLM can be used to extract the grain core conductivity $\left(1 / R_{2}^{\mathrm{pc}}\right)$ and "specific" grain boundary conductivity, as shown in Eq. 11, where the $R$ and $C$ values have been corrected for geometry and porosity.

$\sigma_{\mathrm{gb}}=\frac{\varepsilon_{\mathrm{gb}}}{\varepsilon_{\mathrm{gc}}} \frac{\left(R_{2}^{\mathrm{pc}} C_{2}^{\mathrm{pc}}\right)}{\left(R_{1}^{\mathrm{pc}} C_{1}^{\mathrm{pc}}\right)} \sigma_{\mathrm{gc}} \approx \frac{\left(R_{2}^{\mathrm{pc}} C_{2}^{\mathrm{pc}}\right)}{\left(R_{1}^{\mathrm{pc}} C_{1}^{\mathrm{pc}}\right)} \sigma_{\mathrm{gc}}$

Note that the BLM assumes identical values for grain boundary and grain core dielectric constant $\left(\varepsilon_{\mathrm{gb}}=\varepsilon_{\mathrm{gc}}\right)$.

\section{Results and discussion}

Figure 1 displays the real dielectric constant versus temperature for the YSZ single crystal. For this plot, the dielectric constant was determined from the fitted $C$ value of the arc in the Nyquist plot. Very little arc depression was observed in these plots. At the highest temperatures (dashed line), corrections were made for stray inductive contributions at high frequencies [22]. At room temperature, the value of $\sim 26$ falls within the range of published data for YSZ of comparable composition (23-39) [23-28]. From room temperature to $200{ }^{\circ} \mathrm{C}$, the values obtained are similarly in good agreement with data for YSZ single crystals of slightly higher yttria content (14 mol\%) [29]. The marked increase in dielectric constant between 150 and $250{ }^{\circ} \mathrm{C}$ is most likely associated with the onset of dipolar relaxation. Point defect associates such as $\left(\mathrm{Y}_{\mathrm{Zr}}{ }^{\prime} \mathrm{V}_{\mathrm{O}}^{\bullet \bullet}\right)^{\bullet}$ have been hypothesized, and $\left(\mathrm{Y}_{\mathrm{Zr}}{ }^{\prime} \mathrm{V}_{\mathrm{O}}^{\bullet \bullet} \mathrm{Y}_{\mathrm{Zr}}\right)^{x}$ has been found in $9 \mathrm{~mol} \%$ YSZ [1]. Computational and thermodynamic studies have shown that oxygen vacancies are more likely to sit nearest neighbor to $\mathrm{Zr}$ and second nearest neighbor to $\mathrm{Y}$, and they cluster as third nearest neighbors with other oxygen vacancies along $\langle 111\rangle$ directions [30]. Below $150{ }^{\circ} \mathrm{C}$, both the yttrium acceptors and the oxygen vacancies in associates are immobile. By $250{ }^{\circ} \mathrm{C}$, the oxygen vacancies remain associated, but can hop around their yttrium neighbors, leading to an increase in dielectric response. The data of Fig. 1 were employed for the extraction of local electrical properties using the n-GCM methodology.

Figure 2 displays XRD scans for nanocrystalline samples that were partially sintered at different temperatures for $1 \mathrm{~h}$. Samples sintered at lower temperatures exhibit characteristically large peak broadening, commensurate with the smaller grain sizes. In general, peak broadening at the smallest grain sizes makes it difficult to

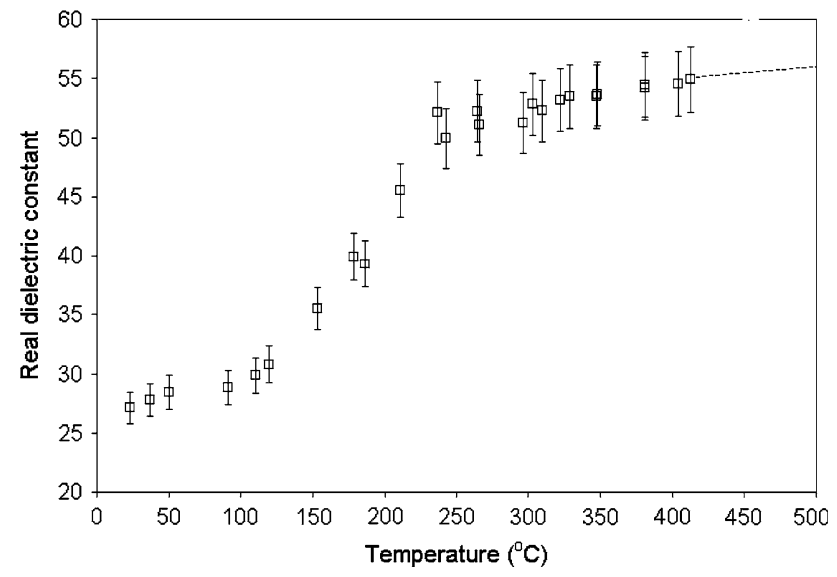

Fig. 1 Real dielectric constant, determined from fitted capacitance values, over a range of temperatures for YSZ single crystals containing $7 \mathrm{~mol} \% \quad \mathrm{Y}_{2} \mathrm{O}_{3}$. The dashed line at higher temperatures indicates values obtained if corrections for stray inductance at high frequencies are incorporated

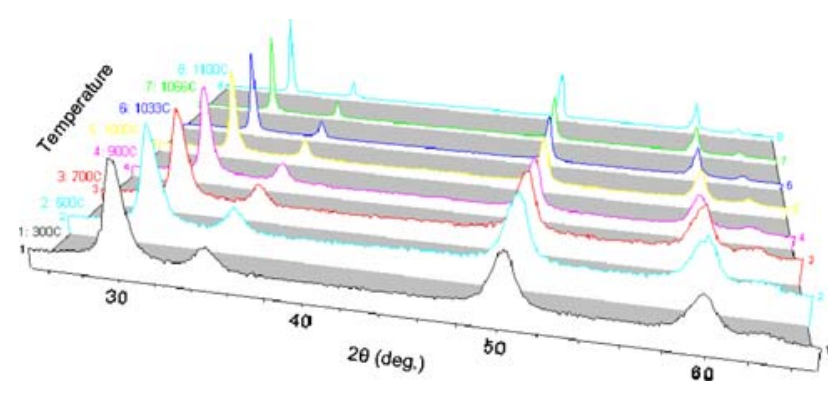

Fig. 2 XRD scans of pellets partially sintered at different temperatures for $1 \mathrm{~h}$ to control grain growth. Broader peaks correspond to smaller grain sizes

determine whether any tetragonal phase is present or if all the peaks correspond to the cubic phase; however, larger grained samples showed no evidence of tetragonal peak splitting.

Figure 3 shows the effect of sintering temperature $(1 \mathrm{~h}$ duration) on grain size and the relationship between grain size and density (the inset diagram). Below $1,000{ }^{\circ} \mathrm{C}$, the samples densify only slightly, with very little detectable grain growth. Between 1,000 and $1,100{ }^{\circ} \mathrm{C}$, there is a dramatic increase in both density (from $40 \%$ to more than $80 \%$ ) and grain size (from $\sim 20 \mathrm{~nm}$ to $\sim 200 \mathrm{~nm}$ ). The average grain sizes of pellets employed for AC-IS measurements, according to the XRD measurements, were 10 , 26, 41, 50, 61, and $73 \mathrm{~nm}$.

Figure 4 shows typical Nyquist plots (for the specimen with an average grain size of $50 \mathrm{~nm}$ ), corrected for geometry but not for porosity. Dual-arc behavior was seen at all temperatures, as evidenced by the data at $362{ }^{\circ} \mathrm{C}$. The thermally activated character of the resistances $\left(R_{1}, R_{2}\right)$ is seen in the dramatic reduction in arc diameters with increasing temperature. It should be remarked that arc 


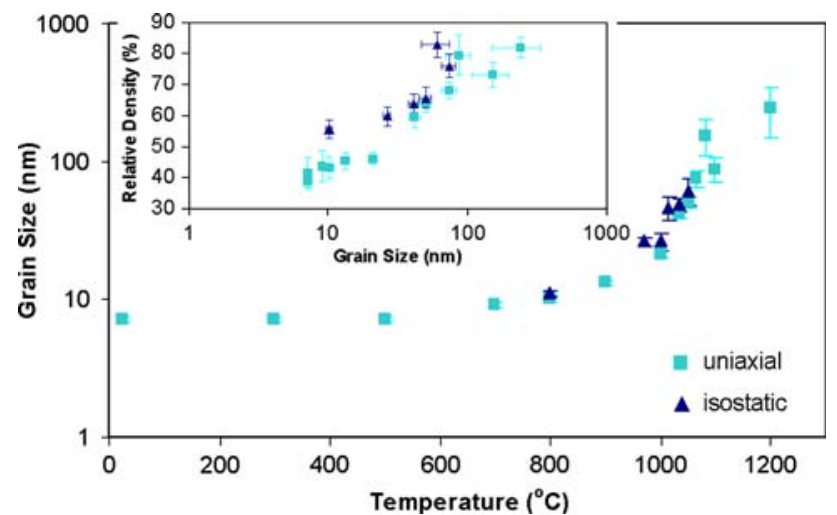

Fig. 3 Grain size as a function of sintering temperature $(1 \mathrm{~h}$ duration) for samples that were only uniaxially pressed (125 MPa) and samples that were isostatically pressed after uniaxial pressing $(125 ; 280 \mathrm{MPa})$. The inset shows the relative density as a function of the grain size, which was incorporated into the porosity correction

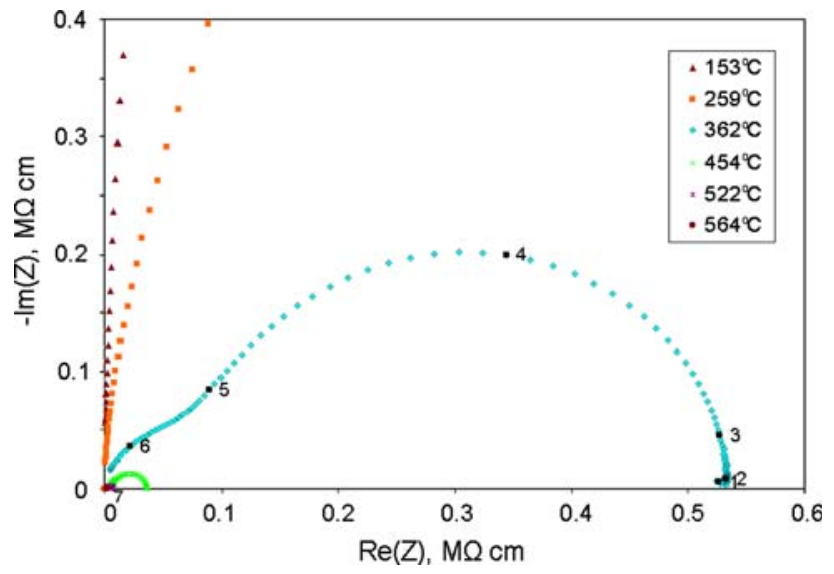

Fig. 4 Impedance spectra for a sample with an average grain size of $50 \mathrm{~nm}$ at different temperatures. Markers correspond to the base 10 logarithm of the linear frequency at that point

convolution became more pronounced as the grain size decreased.

The first step in n-GCM analysis is a plot of predicted dielectric constant versus grain core volume fraction, based upon Eq. 10 and values of $C_{1}$ and $C_{2}$ obtained from fitting the corresponding Nyquist plot. Figure 5 illustrates this procedure for the $10 \mathrm{~nm}$ grain size specimen at $324{ }^{\circ} \mathrm{C}$ $(597 \mathrm{~K})$. If we assume that the grain core dielectric constant must be the same as the single crystal value at the corresponding temperature in Fig. 1, allowing for an estimate of $\pm 10 \%$ uncertainty in the fitted $C_{1}$ and $C_{2}$ values, we arrive at an estimate for the grain core volume fraction of $\phi=0.23 \pm 0.01$ (see Fig. 5a). Similarly, based upon the value of $\phi$, we can estimate values of the grain boundary dielectric constant, grain core conductivity, and grain boundary conductivity (see Fig. 5b). Each of these parameters will be discussed separately.
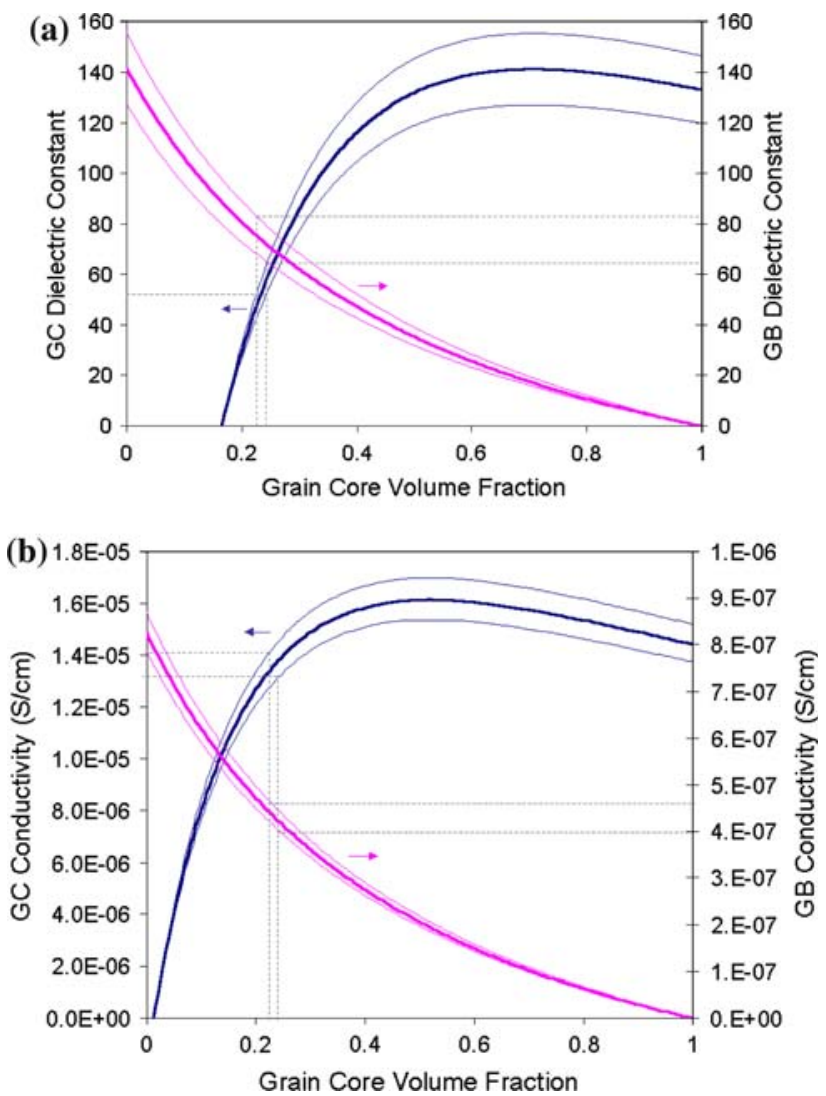

Fig. 5 (a) Calculated grain core and grain boundary dielectric constant versus grain core volume fraction for the $10 \mathrm{~nm}$ sample at $597 \mathrm{~K}$, using Eqs. 9 and 10 and the fitted circuit parameters $\left(C_{1}\right.$ and $C_{2}$ with $10 \%$ error). From a known single crystal (grain core) dielectric constant at the same temperature, the grain core volume fraction and grain boundary dielectric constant may be obtained. (b) Again using the same grain core volume fraction, the grain core and grain boundary conductivities may be obtained. This plot applies to the $10 \mathrm{~nm}$ sample at $597 \mathrm{~K}$ and is calculated from the fitted circuit parameters $\left(R_{1}\right.$ and $R_{2}$ with $5 \%$ error), using Eqs. 7 and 8 . The procedure must be repeated for each temperature and each grain size

Once the volume fraction of grain cores is known, an apparent grain boundary thickness can be calculated assuming a simplified geometry (i.e., the 3D-BLM). Half of this value would correspond to the space charge width, which can be compared with literature values. Table 1 shows half the apparent grain boundary thickness versus grain size for the nanocrystalline specimens. Values are typically $2-4 \mathrm{~nm}(1.84-4.37 \mathrm{~nm})$ for all grain sizes analyzed by the n-GCM. Such values are comparable to space charge widths reported in the literature. Ikuhara et al. [31] showed by energy-dispersive $\mathrm{X}$-ray spectroscopy that in $2.5 \mathrm{~mol} \%$ YSZ, yttrium segregated to within 2-4 nm of the grain boundary core. Other BLM-based calculations yield values around $5 \mathrm{~nm}$ (see [1] and the references therein). (It should be noted, however, that the BLM calculations are only valid at the microscale and employ the assumption that the grain core and grain boundary dielectric constants 
Table 1 Space charge widths (one-half the apparent grain boundary width) for samples with different average grain sizes

\begin{tabular}{ll}
\hline Average grain size $(\mathrm{nm})$ & Half of apparent GB width $(\mathrm{nm})$ \\
\hline 10 & $1.84(1.79-1.88)^{\mathrm{a}}$ \\
26 & $2.08(1.74-2.23)$ \\
35 (dense) & $3.49(3.34-3.61)$ \\
41 & $4.06(3.89-4.20)$ \\
50 & $4.37(4.13-4.53)$ \\
61 & $3.11(2.52-3.52)$ \\
\hline
\end{tabular}

${ }^{a}$ Ranges account for $10 \%$ error in the fitted capacitance values, $C_{1}$ and $C_{2}$, but do not account for the range of grain sizes present in each sample nor for any error in the grain size determination

are equal.) The excellent review by Guo and Waser [1] provides a more detailed explanation of space charge effects in doped zirconia.

Figure 6 displays the grain boundary dielectric constant as a function of temperature for two partially sintered nanocrystalline samples (10 and $41 \mathrm{~nm}$ average grain sizes) and for a dense sample ( $>96 \%$ density) with an average grain size of $35 \mathrm{~nm}$. The dense sample was consolidated by spark plasma sintering and subsequently annealed in air (data provided by co-author Kim) [32]. In general, there is no clear trend with grain size, but values for all the samples are equal to or greater than the single crystal values at the corresponding temperatures (also shown in the figure). There are at least two possible explanations for the large values. First, these data lie above the onset temperature of dipolar relaxation, as seen in the single crystal data of Fig. 1. Therefore, their lower limit is expected to be $\sim 50$, as observed. Second, the dipolar response appears to be enhanced compared to that of the bulk, suggesting that more dipoles (associates) are present at/near the grain boundary. There is evidence from the literature that the ratio of yttrium-to-zirconium ions in the

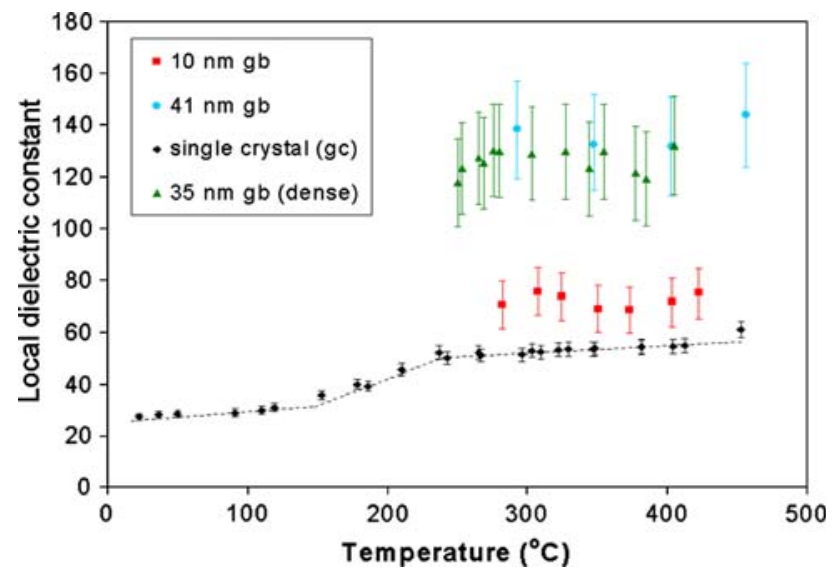

Fig. 6 Grain boundary dielectric constant for samples with average grain sizes of $10 \mathrm{~nm}, 35 \mathrm{~nm}$ (dense), and $41 \mathrm{~nm}$. The single crystal (grain core) dielectric constant is also shown for comparison grain boundary core is significantly enhanced versus the bulk [33]. Additionally, there is evidence of enhanced oxygen vacancy populations in the grain boundary core (see [1] and references therein). Further evidence for higher grain boundary dielectric constants comes from magnetron-sputtered YSZ thin films with a nanoscale columnar microstructure [34]; through-film dielectric constants were consistently higher than the values in Fig. 1 at all temperatures and frequencies, which could be explained on the basis of grain boundaries having dielectric constants larger than that of the grain cores.

The local conductivity values extracted from the AC-IS data via the n-GCM method are plotted in Fig. 7. Figure 7a displays the local grain core and grain boundary conductivities for a partially sintered sample with an average grain size of $26 \mathrm{~nm}$, and for a dense sample of comparable composition (data provided by co-author Kim) with an average grain size of $35 \mathrm{~nm}$. The good agreement between the dense and porous samples for both the local grain core
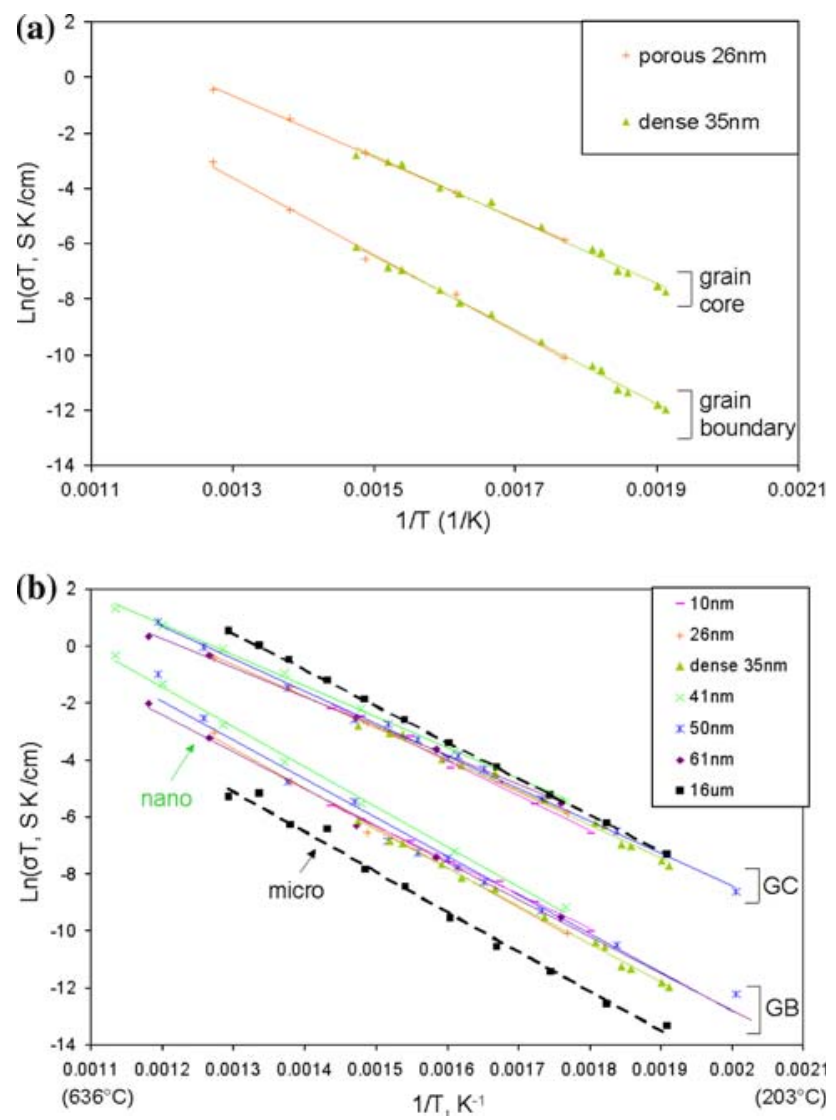

Fig. 7 (a) Local grain core and grain boundary conductivities, calculated using the n-GCM technique, for porous (26 nm grain size) and dense (35 $\mathrm{nm}$ grain size) nanocrystalline samples, showing good agreement after the porosity correction (see text). (b) Local grain core (GC) and grain boundary (GB) conductivities for nanocrystalline and microcrystalline samples (dashed line: $16 \mu \mathrm{m}$ literature data [35]), showing an enhancement in the local grain boundary conductivity in nanocrystalline samples 
and grain boundary conductivities suggests that the porosity correction used in this approach is reasonable.

Figure $7 \mathrm{~b}$ compares the conductivities of microcrystalline and nanocrystalline samples for all the grain sizes studied. The grain core conductivities lie in a narrow band slightly below that of a representative microcrystalline specimen $(16 \mu \mathrm{m}$ grain size, Guo et al. [35] - the upper dashed line). These results suggest that nanocrystalline specimens exhibit a slightly lower grain core conductivity vis-à-vis the microcrystalline specimens. There are several possible explanations for such an effect, including grain size-dependent impurity segregation and possible de-doping of the major acceptor impurity (yttrium) in grain cores as the grain size decreases [1].

The grain boundary conductivities of the nanocrystalline specimens fall in a narrow band lying significantly above the data for the representative microcrystalline specimen (Guo et al. [35] — dashed line). For the microcrystalline data, the specific grain boundary conductivity is plotted, which was obtained from the BLM (Eq. 11), assuming $\varepsilon_{\mathrm{gb}}=\varepsilon_{\mathrm{gc}}$. For the nanocrystalline specimens, the local grain boundary conductivities are plotted, as obtained by n-GCM analysis. (This comparison of local and specific conductivities uses the assumption of nearly isotropic grain boundary transport, i.e., that conductivity is not substantially different perpendicular versus parallel to the grain boundary. This limitation is discussed further later in the article.) In this case, the enhancement of grain boundary conductivity is significant, in agreement with prior reports in the literature $[1,4]$.

There are several feasible explanations for increases in the grain boundary conductivity of the nanocrystalline samples. One possibility involves a decrease in the positive space charge potential at the grain boundary core with decreasing grain size [1]. Figure 8 shows the calculated grain boundary potential (relative to the grain core potential) as a function of grain size. The potential was calculated from the local grain core and grain boundary conductivities, using an equation developed by Fleig et al. [36] for a double Schottky barrier:

$\frac{\sigma_{\mathrm{gc}}}{\sigma_{\mathrm{gb}}}=\frac{\exp \left(\frac{z e \Delta \varphi(0)}{k T}\right)}{\frac{2 z e \Delta \varphi(0)}{k T}}$

where $z$ is the relative charge of the current-carrying defect in the lattice ( $z=2$ for oxygen vacancies), $e$ is the electronic charge, and $\Delta \varphi(0)$ is the potential of the grain boundary core relative to the grain cores.

As can be seen from Fig. 8, the calculated grain boundary potential decreases monotonically with decreasing average grain size. This result would suggest that nanocrystalline samples have higher concentrations of oxygen vacancies in the grain boundaries than their microcrystalline

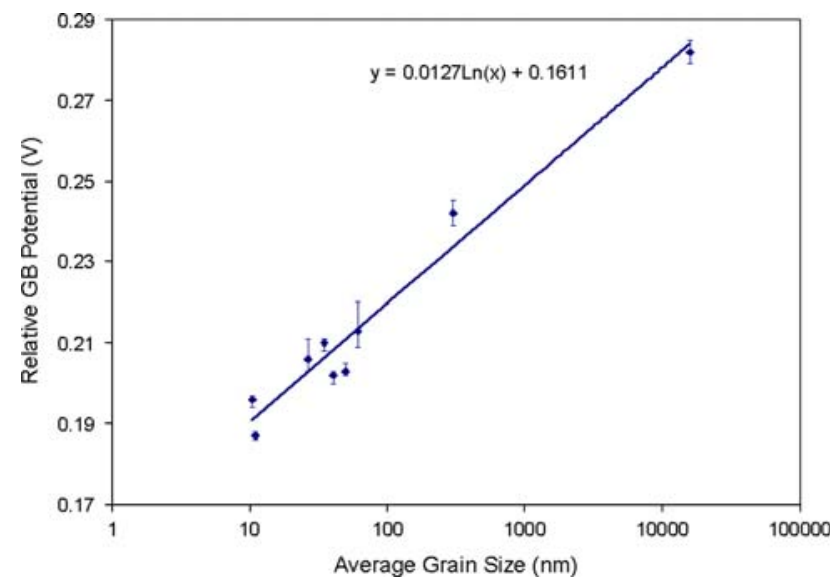

Fig. 8 Grain boundary potential (relative to grain core potential) calculated using Eq. 12 for samples with different average grain sizes in the temperature range $619-636 \mathrm{~K}$. Conductivities for the $16 \mu \mathrm{m}$ sample, used in the calculation, were taken from [35]

counterparts, even though oxygen vacancies are still depleted within the grain boundaries relative to the grain interiors. This increase in carrier concentration could explain the improvement in the grain boundary conductivity. However, it should be pointed out that this explanation is usually reserved for high purity specimens [1].

An alternate explanation involves grain size-modified segregation, with dilution of the impurities that segregate to grain boundaries in nanocrystalline specimens. This effect is due to the dramatic increase in grain boundary area (in nanograined samples) over which to distribute such segregating impurities. In other words, the grain boundaries are actually cleaner in nanocrystalline versus microcrystalline specimens.

In Fig. 9, the total conductivity is plotted for samples with different average grain sizes. The total conductivity was taken as the inverse of the total resistance $\left(R_{1}+R_{2}\right)$, after correction for geometry and porosity. From this

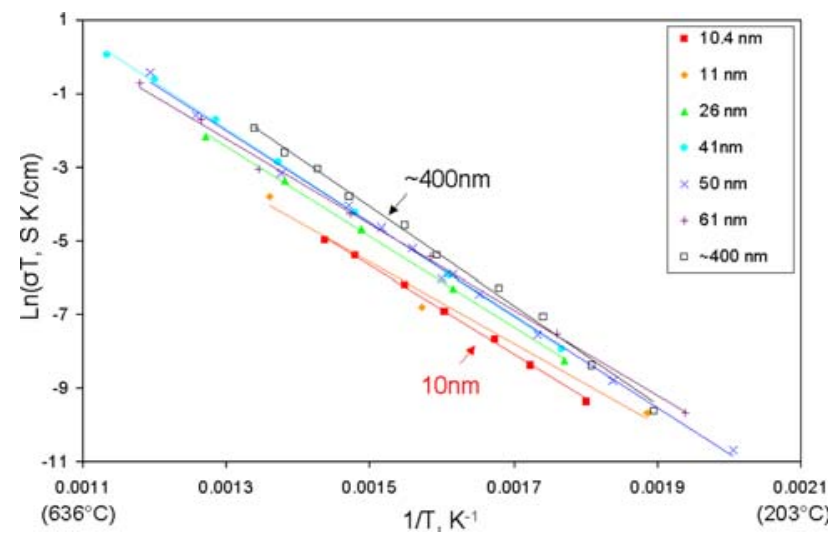

Fig. 9 Total ionic conductivities for a range of grain sizes, after porosity correction. Total conductivity consistently decreases as the average grain size decreases 
figure, it is evident that decreasing the grain size does not improve the total conductivity; rather, the conductivity decreases monotonically with decreasing grain size. This result may seem surprising in light of the enhancement in the grain boundary conductivity in nanocrystalline samples. The decrease in total conductivity, however, can be explained by (1) the observed decrease in grain core conductivity (Fig. 7b) and (2) the dramatic increase in the number of grain boundary barriers opposing transport as grain size is reduced into the nano-regime. In other words, individual grain boundaries may be less resistive in nanoversus micro-crystalline material, but they remain more resistive than grain cores (Fig. 7b) and their increased number swamps out the effect of a marginal increase in local conductivity.

It is worthwhile to note some of the limitations of the n-GCM approach. In theory, the n-GCM should be applicable at all grain sizes (grain core volume fractions). For example, in our prior work [10,11], the n-GCM approach was shown to be in good agreement with the 3D BLM simulation (which included the true current distribution) across the entire range of volume fractions. In reality, the approach will most likely become intractable at grain core volume fractions of 0.9 or larger, owing to experimental uncertainties associated with establishment of reliable grain core fractions (as per the n-GCM procedure demonstrated in Fig. 5a and b). If we arbitrarily assume a space charge width of $2 \mathrm{~nm}$, a grain core volume fraction of 0.9 would be reached at a grain size of $\sim 100 \mathrm{~nm}$. Therefore, we would anticipate a working grain size range for the n-GCM of 10-100 nm.

In reality, the situation can be even further constrained. We have established that the n-GCM requires equiaxed nanostructures with a narrow distribution of grain sizes. In the present study, the AC-IS data for the $73 \mathrm{~nm}$ grain size specimen could not be successfully analyzed by n-GCM; it was not possible to match the measured single crystal dielectric constant in the n-GCM-derived $\varepsilon_{\mathrm{gc}}$ versus $\phi$ plot (e.g., see Fig. 5a). We attribute this limitation to a broad distribution of grain sizes leading to significant arcdepression in AC-IS plots. Of course, better processing resulting in nanostructures with the requisite equiaxed/ monosized grains should extend the applicability of the $\mathrm{n}$-GCM to the $10-100 \mathrm{~nm}$ range of grain size, as described above.

Another limitation of the n-GCM is that it assumes a two-phase microstructure, where grain boundaries and grain cores are considered isotropic and homogeneous. (The n-GCM approach does not explicitly incorporate the gradually changing defect populations or conductivities expected at the grain core-grain boundary interface of real electroceramics. Such behavior has been modeled in preliminary simulations, by nesting multiple grain boundary layers in the 3D-BLM structure [37].) The n-GCM also assumes that conductivity along the grain boundary $\left(\sigma_{\mathrm{gbl}}\right)$ is equal to conductivity across the grain boundary $\left(\sigma_{\mathrm{gb} \perp}\right)$. Nonetheless, the n-GCM can be used to analyze samples with anisotropic grain boundaries if the impedance spectra exhibit dual arc behavior, which is the case as long as $\sigma_{\mathrm{gb} \perp}<\sigma_{\mathrm{gb \|} \|} \ll \sigma_{\mathrm{gc}}$. Equations for the case when $\sigma_{\mathrm{gb} \|}$ is only slightly smaller than $\sigma_{\mathrm{gc}}$ have been provided by Kidner [38].

Finally, the n-GCM requires high quality impedance data with minimal contributions from stray inductive effects or parallel capacitances [22]. Extreme care should be taken to avoid such parasitic contributions by minimizing cable/lead lengths and by employing separate shielded/grounded leads in AC-IS measurements. More information about the n-GCM analysis and its limitations can be found in our recent review [39].

\section{Conclusions}

This paper has demonstrated the applicability of the $\mathrm{n}$-GCM to the determination of local properties (conductivities and dielectric constants) of nanocrystalline YSZ. This model replaces the traditionally used BLM at the smallest grain sizes, and in this case characterized samples with average grain sizes between 10 and $73 \mathrm{~nm}$. In order to apply the model, AC-IS was carried out on nanocrystalline and microcrystalline samples over a range of temperatures. The dielectric constant of a single crystal of a similar dopant concentration was also determined over the same temperature range for use in the analysis as the grain core dielectric constant. The sharp increase in single crystal dielectric constant with temperature was attributed to the onset of dipolar relaxation. The grain boundary dielectric constants were consistently higher than the single crystal (grain core) dielectric constant, likely due to an enhanced dipolar response in the disordered grain boundaries, consistent with the enhancement of certain defect populations and/or mobilities in the grain boundaries. The grain core conductivities were slightly lower in nanocrystalline samples relative to microcrystalline values, but local grain boundary conductivities were significantly enhanced in the nanocrystalline samples, albeit still at levels less than that of the grain cores (i.e., they remain barriers to transport). The enhancement in the local grain boundary conductivity in the nanocrystalline samples is consistent with the observed decrease in grain boundary space charge potential with decreasing grain size, but may be also be attributable to the dilution of impurities at grain boundaries, i.e., grainsize mediated impurity segregation. In spite of the local grain boundary conductivity increase at the nanoscale, the total conductivity decreased with decreasing grain size, 
owing to the increase in the number of blocking grain boundaries in the nanocrystalline specimens.

Acknowledgements The authors acknowledge support from the U.S. Department of Energy under contract no. DE-FG02-05ER-46255 (NHP, TOM) and a National Science Foundation Graduate Research Fellowship (NHP).

Open Access This article is distributed under the terms of the Creative Commons Attribution Noncommercial License which permits any noncommercial use, distribution, and reproduction in any medium, provided the original author(s) and source are credited.

\section{References}

1. Guo X, Waser R (2006) Prog Mater Sci 51:151

2. Fergus JW (2006) J Power Sour 162:30

3. Tang CQ, Liu L, Qian XL, Guo X, Sun YQ, Yao KL, Cui K (1998) Ionics 4:472

4. Mondal P, Klein A, Jaegermann W, Hahn H (1999) Solid State Ionics 118:331

5. Madani A, Cheikh-Amdouni A, Touati A, Labidi M, Boussetta H, Monty C (2005) Sensor Actuat B Chem 109:107

6. Verkerk MJ, Middlehuis BJ, Burggraaf AJ (1982) Solid State Ionics 6:159

7. Kosacki I, Suzuki T, Petrovsky V, Anderson HU (2000) Solid State Ionics 136-137:1225

8. Karthikeyan A, Chang C-L, Ramanathan S (2006) Appl Phys Lett 89:183116

9. Guo X, Vasco E, Mi S, Szot K, Wachsman E, Waser R (2005) Acta Mater 53:5161

10. Kidner NJ, Homrighaus ZJ, Ingram BJ, Mason TO, Garboczi EJ (2005) J Electroceram 14:283

11. Kidner NJ, Homrighaus ZJ, Ingram BJ, Mason TO, Garboczi EJ (2005) J Electroceram 14:293

12. Bauerle JE (1969) J Phys Chem Solid 30:2657

13. Beekmans NM, Heyne L (1976) Electrochim Acta 21:303

14. van Dijk T, Burggraaf AJ (1981) Phys Status Solidi A 63:229
15. Boukamp BA (ㄷ1986-2005) Equivalent circuit for windows, Version 1.2, University of Twente, The Netherlands/WisseQ

16. Näfe H (1984) Solid State Ionics 13:255

17. Hwang J-H, McLachlan DS, Mason TO (1999) J Electroceram 3:7

18. Bonanos N, Lilley E (1981) J Phys Chem Solids 42:943

19. Jade, version 8 , Materials data incorporated (MDI), California, USA

20. Hwang J-H, Kirkpatrick KS, Mason TO, Garboczi EJ (1997) Solid State Ionics 98:93

21. McLachlan DS, Blaszkiewicz M, Newnham RE (1990) J Am Ceram Soc 73(8):2187

22. Edwards DD, Hwang J-H, Ford SJ, Mason TO (1997) Solid State Ionics 99:85

23. Samara GA (1990) J Appl Phys 68:4214

24. Bates JB, Wang JC (1988) Solid State Ionics 28-30:115

25. Zhu J, Liu ZG (2003) Mater Lett 57:4297

26. Thompson DP, Dickins AM, Thorp JS (1992) J Mater Sci 27:2267

27. Chen Y, Sellar JR (1996) Solid State Ionics 86-88:207

28. Subramanian MA, Shannon RD (1989) Mater Res Bull 24(12): 1477

29. Lanagan MT, Yamamoto JK, Bhalla A, Sankar SG (1989) Mater Lett 7(12):437

30. Navrotsky A, Simoncic P, Yokokawa H, Chen W, Lee T (2007) Faraday Discuss 134:171 and references therein

31. Ikuhara Y, Thavorniti P, Sakuma T (1997) Acta Mater 45:5275

32. Lee JS, Anselmi-Tamburini U, Munir ZA, Kim S (2006) Electrochem Solid St 9:J34

33. Lei Y, Ito Y, Browning ND, Mazanec TJ (2002) J Am Ceram Soc 85:2359

34. Boulouz M, Tcheliebod F, Boyer A (1997) J Eur Ceram Soc 17:1741

35. Guo X, Maier J (2001) J Electrochem Soc 148(3):E121

36. Fleig J, Rodewald S, Maier J (2000) J Appl Phys 87:2372

37. Kidner NJ, Ingram BJ, Homrighaus ZJ, Mason TO, Garboczi EJ (2003) Mat Res Soc Proc 756:39

38. Kidner NJ (December 2006) Modeling the electrical (impedance/ dielectric) behavior of nanocrystalline and thin film electroceramics, $\mathrm{PhD}$ dissertation, Northwestern University

39. Kidner NJ, Perry NH, Mason TO, Garboczi EJ (2008) J Am Ceram Soc. doi:10.1111/j.1551-2916.2008.02445.x 